\title{
Acknowledgement to reviewers of Journal of Cancer Metastasis and Treatment in 2019
}

\author{
Journal of Cancer Metastasis and Treatment Editorial Office \\ OAE Publishing Inc., Chaoyang District, Beijing 100016, China.
}

Correspondence to: OAE Publishing Inc., Rm. 968, 9th Floor, Xinhengji International Building, No. 3 Maizidian West Road, Chaoyang District, Beijing 100016, China. E-mail: editorialoffice@jcmtjournal.com

How to cite this article: Journal of Cancer Metastasis and Treatment Editorial Office. Acknowledgement to reviewers of Journal of Cancer Metastasis and Treatment in 2019. J Cancer Metastasis Treat2020;6:2. http://dx.doi.org/10.20517/2394-4722.2020.07

Received: 19 Jan 2020 Accepted: 19 Jan 2020 Published: 21 Jan 2020

Science Editor: Lucio Miele Copy Editor: Jing-Wen Zhang Production Editor: Jing Yu

The editors of the Journal of Cancer Metastasis and Treatment (JCMT) would like to take this opportunity to express their sincere gratitude to the reviewers and guest editors for assessing manuscripts in 2019 [Table 1].

Table 1. Names of reviewers and guest editors

\begin{tabular}{llll}
\hline Names & & & \\
\hline Albig, Allan & Gewirtz, David A. & Liu, Shanrong & Siravegna, Giulia \\
Anders, Carey & Gollahon, Lauren S. & Liu, Tingjiao & Skvortsova, Ira-Ida \\
Anfossi, Simone & Goossens, Steven & Lu, Renquan & Socha, Joanna \\
Arendt, Lisa M. & Grivicich, Ivana & Luwor, Rodney Brian & Sossey-Alaoui, Khalid \\
Argyle, David & Gunjan, Akash & Lyons, Traci & Sotelo, Julio \\
Azab, Kareem & Guo, Peixuan & Marino, Maria & Sriuranpong, Virote \\
Bai, Mingfeng & Hamilton, Gerhard & Martínez-Ordóñez, Anxo & Suami, Hiroo \\
Baxevanis, Constantin N. & Holland, John & Morgan, Maria & Swamynathan, Shivalingappa K. \\
Bazin, Dominique C. & Howe, Philip & Morgese, Francesca & Szabo, Eva \\
Berardi, Rossana & Hudson, Barry & Mrugala, Maciej & Szewczuk, Myron R. \\
Bhatt, Aashish & Huo, Dezheng & Nagaraju, Ganji Purnachandra & Teixeira, Ana \\
Bhavsar, Amit P. & Huycke, Mark & Nakao, Mitsuyuki & Tham, Tristan \\
Bischetti, Simone & lorio, Egidi & Nawrocki, Sergiusz & Thomas, Clement \\
Bonetto, Andrea & Isidoro, Ciro & Okami, Kenji & Thomson, Timothy M. \\
Bown, Stephen G. & Jaggi, Meena & Okines, Alicia F.C. & Tomasetti, Marco \\
Brodsky, Leonid & Johnson, Daniel E. & Osella-Abate, Simona & Torniai, Mariangela \\
Brooks, George A. & Johnson, Rachelle W. & Pajtler, Kristian & Tuccari, Giovanni \\
Burnstein, Kerry L. & Jolly, Mohit Kumar & Park, Jonghoon & Turner, Nigel \\
Chang, Kwang-Yu & Karamouzis, Michalis & Pazienza, Valerio & Vaidya, Shantashri
\end{tabular}

Cc) (i) (c) The Author(s) 2020. Open Access This article is licensed under a Creative Commons Attribution 4.0 , adaptation, distribution and reproduction in any medium or format, for any purpose, even commercially, as long as you give appropriate credit to the original author(s) and the source, provide a link to the Creative Commons license, and indicate if changes were made. 


\begin{tabular}{llll} 
Chen, Dongquan & Karsdal, Morten & Péchoux, Cécile & van den Berg, Anke \\
Chen, Pai-Sheng & Kato, Yasumasa & Penna, Fabio & van Norren, Klaske \\
Cheung, Chun Hei Antonio & Ke, Zunfu & Peters, Godefridus J. & Varadan, Vinay \\
Chowdhury, Imran & Keshamouni, Venkat & Pisanu, Maria Elena & Vetto, John T. \\
Christophi, Christopher & Kim, Chae-Yong & Porter, Weston & Videira, Paula \\
de Amorim, MariaGalli & Kimple, Randall & Potdar, Pravin D. & Wagner, Kay-Uwe \\
De Wagter, Carlos & Kniss, D. A. & Prosperi, Jenifer R. & Wang, Bingcheng \\
Demonacos, Constantinos & Kovacevic, Zaklina & Raffa, Vittoria & Wasilewska-Tesluk, Ewa \\
Deppert, Wolfgang & Kratochwil, Clemens & Ralph, Stephen & Wei, Dao-yan \\
Dittmer, Jürgen & Kwok, Hang Fai & Reagan, Michaela & Wendt, Mlchael \\
Djamgoz, Mustafa B.A. & Lam, Eric W.-F. & Ricciardelli, Carmela & Werner-Wasik, Maria \\
Dorfman, Ruslan & Lannin, Donald & Richer, Jennifer & Wrona, Anna \\
Fang, Bingliang & Lappano, Rosamaria & Sacco, Antonio & Wu, Yadi \\
Fedele, Monica & Lee, Choonik & Saha, Achinto & Würth, Roberto \\
Ferrari, Paola & Lee, Minhyung & Sankhwar, Satya Narayan & Xavier, Jennifer \\
Frassanito, Maria Antonia & Leichtle, Alexander Benedikt & Schiemann, William & Xi, Yaguang \\
Fujino, Hiromichi & Lemos, Henriquede Paula & Schneider, Sallie S. & Xu, Chuanshan \\
Gaidano, Gianluca & Li, Zhaoyu & Schuller, Hildegard & Xu, Ren \\
Gambari, Roberto & Liao, Shuen-Kuei & Shin, Vivian Yvonne & Yang, Shihan \\
Gao, Alan & Lin, Hongyu & Shivapurkar, Narayan & Yang, Yong \\
Gao, Yenan & Liu, Huiyu & Siddiqui, Rafat A. & Yazdanparast, Razieh \\
\hline
\end{tabular}

The listed reviewers have our sincere gratitude. They can also get their Reviewer Recognition Certificates by building their peer review profiles on Publons (https://publons.com).

If you are interested in becoming a reviewer for JCMT, you are welcome to apply at https://jcmtjournal. com/journal/vol_reviewer.

\section{DECLARATIONS}

\section{Authors' contributions}

Writing and revision of the article and approved the final version: Journal of Cancer Metastasis and Treatment Editorial Office.

\section{Availability of data and materials}

Not applicable.

\section{Financial support and sponsorship}

None.

\section{Conflicts of interest}

All authors declared that there are no conflicts of interest.

\section{Ethical approval and consent to participate}

Not applicable.

\section{Consent for publication}

Not applicable.

\section{Copyright}

(c) The Author(s) 2020. 